\title{
A test of constancy of dark matter halo surface density and radial acceleration relation in relaxed galaxy groups
}

\author{
Gopika K.* and Shantanu Desai ${ }^{\dagger}$ \\ Department of Physics, Indian Institute of Technology, Hyderabad, Telangana-502285, India
}

\begin{abstract}
The dark matter halo surface density, given by the product of the dark matter core radius $\left(r_{c}\right)$ and core density $\left(\rho_{c}\right)$ has been shown to be a constant for a wide range of isolated galaxy systems. Here, we carry out a test of this ansatz using a sample of 17 relaxed galaxy groups observed using Chandra and XMM-Newton, as an extension of our previous analysis with galaxy clusters. We find that $\rho_{c} \propto r_{c}^{-1.35_{-0.17}^{+0.16}}$, with an intrinsic scatter of about $27.3 \%$, which is about 1.5 times larger than that seen for galaxy clusters. Our results thereby indicate that the surface density is discrepant with respect to scale invariance by about $2 \sigma$, and its value is about four times greater than that for galaxies. Therefore, the elevated values of the halo surface density for groups and clusters indicate that the surface density cannot be a universal constant for all dark matter dominated systems. Furthermore, we also implement a test of the radial acceleration relation for this group sample. We find that the residual scatter in the radial acceleration relation is about 0.32 dex and a factor of three larger than that obtained using galaxy clusters. The acceleration scale which we obtain is in-between that seen for galaxies and clusters.
\end{abstract}

\section{INTRODUCTION}

In a recent work [1] (GD20, hereafter), we carried out an observational test using a sample of 12 relaxed galaxy clusters observed using the Chandra X-ray observatory [2], to ascertain if the dark matter halo surface density is constant. This was motivated by the claim from some groups that the dark matter halo surface density has been observed to be invariant, for a wide variety of systems spanning over 18 orders in blue magnitude, for a diverse suite of systems from spiral galaxies to dwarf galaxies [3-6]. This dark halo surface density $\left(S_{\text {surf }}\right)$ is given by the product of core radius $\left(r_{c}\right)$ and core density $\left(\rho_{c}\right)^{1}$.

$$
S_{\text {surf }}=\rho_{c} \times r_{c}
$$

Both $r_{c}$ and $\rho_{c}$ were obtained by fitting a Burkert [7] or other cored profiles, or in a model-independent fashion as done in GD20 or [8]. The current best-fit values for the dark matter surface density for single galaxy systems is given by $\log \left(\rho_{c} r_{c}\right)=(2.15 \pm 0.2)$ in units of $M_{\odot} p c^{-2}$ [6].

The main premise behind the above results is that all dark matter profiles are cored. However, the cored profiles do not provide a pristine fit to all types of systems from dwarf galaxies to clusters, some of which need cuspy profiles [9-14]. Therefore, this result has been disputed by other authors, who argue that the halo surface density is correlated with the luminosity, mass, and other galaxy properties [15-21].

Given these conflicting results, a further test of the constancy of dark matter halo surface density for a large suite of astrophysical systems would be an acid test for

\footnotetext{
*E-mail:ph19resch01001@iith.ac.in

${ }^{\dagger}$ E-mail: shntn05@gmail.com

${ }^{1}$ Note that $\rho_{c}$ is also sometimes referred to as central density [4]
}

$\Lambda \mathrm{CDM}$ as well as various alternatives. For example, it has been shown recently that the observed constant surface density is in tension with predictions from fuzzy dark matter models, thus ruling them out [22]. A recent recap of the predictions of some of the myriad theoretical scenarios for the halo surface density can be found in GD20. Prior to GD20, there was only one work [8], which implemented this test for a large sample of galaxy clusters and showed that the power-law exponent for the relation between $\rho_{c}$ and $r_{c}$ ranges from -1.46 to -1.6 , instead of -1 , which is expected for a constant halo density. GD20 then showed using the Chandra cluster dataset that $\rho_{c} \propto r_{c}^{-1.08 \pm 0.55}$, indicating that the halo surface density is almost invariant. GD20 showed that they could reproduce the earlier result [8] for the Chandra dataset by neglecting the stellar and gas mass contribution to the total mass, similar to the analysis in [8]. However, the observed dark matter halo surface density in GD20 (and also [8]) is about an order of magnitude higher $\left(\log \left(r_{c} \rho_{c}\right) \sim 3\right.$ in units of $\left.M_{\odot} p c^{-2}\right)$ than what has been deduced for single galaxy systems [6]. Therefore, at face value this clearly shows the dark matter surface density of haloes cannot be universal (constant) at all scales, as claimed in [3].

It is still an open question, on whether the elevated halo surface density for galaxy clusters is consistent with hydrodynamical simulations of $\Lambda \mathrm{CDM}$ and alternatives [1]. Therefore, given the observed increase in surface density for cluster scale haloes compared to galaxies, it is imperative to carry out this test for astrophysical systems in an intermediate mass range, which bridge the gap between single galaxies and clusters. Groups provide the ideal laboratory for this purpose.

Another intriguing observational result was found by $[23,24]$, who obtained a pristine deterministic relation between the baryonic $\left(a_{b a r}\right)$ and total acceleration $\left(a_{t o t}\right)$ from spiral galaxies in the SPARC galaxy sample, with the scatter attributed to observational uncertainties. A 
similar relation has also been observed for elliptical galaxies [25]. This has been dubbed as Radial Acceleration Relation (RAR).

$$
a_{t o t}=\frac{a_{b a r}}{1-e^{-\sqrt{a_{b a r} / a_{0}}}},
$$

where $a_{0} \sim 1.2 \times 10^{-10} \mathrm{~m} / \mathrm{s}^{2}$ [23]. More generally, this relation can be recast as a linear regression between $a_{t o t}$ and $a_{b a r}$ in log-log space [26, 27]. A few groups have disputed the existence of this acceleration scale using the same data or found higher scatter using other samples [28-31]. Nevertheless, this relation can be shown to be a trivial consequence of the MOND paradigm [32]. There are conflicting results in literature on whether $\Lambda \mathrm{CDM}$ simulations and semi-analytical models can reproduce the acceleration scale and the tight scatter (See [33] for an up-to-date recap of most of these predictions). Most recently, [33] has shown that they can reproduce the observed scatter in the RAR from quasi-adiabatic relaxation of dark matter haloes in the presence of baryons. Although, it is known for more than three decades that the MOND phenomenology cannot obviate the need for dark matter in galaxy clusters [34, 35], a test of RAR would be an acid test for the models which reproduce MOND behavior at galactic scales but need dark matter at cluster scales. Motivated by these considerations, multiple groups $[26,27,36,37]$ carried out a test of the RAR using different samples of galaxy clusters. These analyses found a tight scatter of between 0.11 to 0.13 dex and an elevated acceleration scale $a_{0} \sim 10^{-9} \mathrm{~m} / \mathrm{s}^{2}$. This acceleration scale is again about an order of magnitude larger than that seen for galaxies in the SPARC sample.

As a follow-up to these sets of tests, we now carry out a test of both a constant dark matter halo surface density and RAR for galaxy groups. Galaxy groups are less massive, gravitationally bound systems compared to galaxy clusters, with intra-group temperatures less than $2 \mathrm{keV}[38,39]$. However, they cannot be considered as scaled down versions of massive clusters, only because of their shallow gravitational potential. Groups are much more susceptible to complex baryonic physics. For these reasons, groups have proved to be excellent laboratories for understanding the baryonic physics needed for understanding galaxy formation [38, 40-43].

Although, MOND has been tested (and disfavored) with X-ray selected relaxed groups [44], there has been no test of RAR or constant halo surface density for galaxy groups. Since galaxy clusters point to an acceleration scale and halo surface density, about an order of magnitude higher than for galaxies $[1,36]$, galaxy groups provide the ideal dataset to bridge the gap between the two. Combining the surface density data of groups with cluster enables us to increase the dynamic range to test the scaling with $M_{200}$, as predicted in some theoretical models $[18,45]$.

This manuscript is structured as follows. We describe the G07 group sample and associated models for the mass and acceleration profile in Section II. Our analysis and results for the relation between core radius and density and the radial acceleration relation (RAR) can be found in Section III and Section IV, respectively. We also test for a dependence of the dark matter scale parameters with mass and luminosity in Section V. Our results on the dark matter column density (another variant of the surface density) are presented in Section VI. We conclude in Section VII.

\section{DATA SAMPLE}

We use X-ray observations of 17 galaxy groups with redshifts upto $z=0.08$ from Gastaldello et al. (G07) and Zappacosta et al. (Z06) for this work. These groups were imaged with Chandra and/or XMM-Newton. The Chandra satellite has a high spatial resolution, which helps in resolving the temperature and density profiles in the cores. On the other hand, the high sensitivity of XMM-Newton provides very good signal-to-noise $(\mathrm{S} / \mathrm{N})$ ratios in the outer regions. Their exposure times ranged from 10-75 ks. These groups with masses $\left(10^{13}-10^{14} M_{\odot}\right)$ span the range between galaxies and clusters, with temperatures between 1-3 keV. The groups were imaged upto outer radii of $730 \mathrm{kpc}$ (cf. Table 1 in G07). More details of the observations and data reduction can be found in the aforementioned works $[46,47]$. The dataset in G07 and Z06 consists of the brightest and the most relaxed groups, which were selected in order to derive robust constraints on the mass profiles. This allows us to apply the equation of hydrostatic equilibrium to obtain the masses. The only exception is RGH 80, which has been proposed to be a sub-merging group [48]. In G07, it has been included as it is part of a complete X-ray flux limited sample observed by Chandra and so that it can be used for comparing against the very relaxed systems. The X-ray brightness and temperature was fitted with 3-D parametric models described in G07. More detailed description about these parametric gas density and temperature models for this sample of galaxy groups can be found in G07 and Z06.

\section{A. Mass and Density in Groups}

As the groups we choose for this analysis are relaxed systems, we can apply the equation of hydrostatic equilibrium in these systems in order to determine their total gravitating mass. Errors due to hydrostatic equilibrium assumption could contribute a systematic error of upto $\sim 15 \%$ [49]. Hence, the total mass of the galaxy groups can be derived, given the temperature and gas density models and also assuming an ideal gas equation of state 
as [50]

$$
M(r)=-\frac{k T(r) r}{G \mu m_{p}}\left(\frac{d \ln \rho_{g}}{d \ln r}+\frac{d \ln T}{d \ln r}\right)
$$

The 3-D temperature and gas density data were obtained by fitting the X-ray data with the parametric models available in G07. These temperature and gas density data thus obtained were used to derive the mass using Eq. (3). The temperature and density points at various radii for each of these groups were made available to us (F. Gastaldello, private communication). We used a spline interpolation to determine the derivatives of the logarithm of the gas density and temperature needed to evaluate Eq. 3. The only exception was A2589, for which we used the best-fit values of the parametric models available in Z06, to derive the temperature and gas density profiles, and thereby the mass.

Once we have the total mass, we then subtract the baryonic mass in order to obtain the dark matter mass:

$$
\begin{aligned}
& M_{D M}=M(r)-M_{\text {baryon }} \\
\text { where, } & M_{\text {baryon }}=M_{\text {gas }}+M_{B C G}
\end{aligned}
$$

Here, $M_{g a s}$ and $M_{B C G}$ denote the mass of the gas and Brightest Cluster galaxy (BCG) respectively. The gas mass can be obtained from the gas density assuming spherical symmetry:

$$
M_{g a s}=\int 4 \pi r^{2} \rho_{g}(r) d r
$$

For most of the groups in this sample, the stellar contribution from the non-central galaxies is below $10 \%$ except for AWM 4 and A262. In order to account for the stellar counterpart, we subtract a BCG constant mass from the total mass, similar to the analysis in [44]. The mass of the $\mathrm{BCG}$ is determined with the assumption that $M / L_{K}=1$, where $L_{K}$ is the luminosity in the $K$-band. This ansatz is valid for the older stellar populations and a Kroupa IMF ([51]). However, for a Salpeter IMF, $M / L_{k}$ ratio can increase upto 50\% [52]. Note however that in G07, the stellar mass has been modeled using a De Vaucouleurs profile. The $K$-band luminosities for all the groups have been obtained from G07 and [44].

The density of the dark matter halos can be obtained from the mass distribution by positing spherical symmetry:

$$
\rho_{D M}(r)=\frac{1}{4 \pi r^{2}} \frac{d M_{D M}}{d r}
$$

The errors in the temperature and gas density for the selected radii for the groups in G07 were also made available to us,(F. Gastadello, private correspondence) which were used to propagate the errors in mass and density of the dark matter halos. For A2589, the errors were determined by propagating the errors of the best-fit parameters of the temperature and gas density models in Z06. The errors in the spherical symmetry assumption could be up to 5\%, as discussed in GD20 and references therein. These were not included in our error budget. We also do not consider the errors due to the uncertainty in the stellar mass distribution in our analysis.

The dark matter core density $\left(\rho_{c}\right)$ and core radius $\left(r_{c}\right)$ is obtained by fitting the halo density to a Burkert profile $[3,7]$,

$$
\rho(r)=\frac{\rho_{c} r_{c}^{3}}{\left(r^{2}+r_{c}^{2}\right)\left(r+r_{c}\right)}
$$

\section{B. Acceleration Profile in Groups}

In order to test the RAR for groups, we need to determine the total acceleration as well as that due to only the baryonic content at different radii from the center. The total acceleration was synthesized from the total mass (obtained from Eq. 3),

$$
a_{t o t}=\frac{G M(r)}{r^{2}}
$$

Similarly, it is straight-forward to determine the acceleration due to the baryons alone, given the baryonic mass (obtained from Eq. 5). Thus, we have,

$$
a_{\text {baryon }}=\frac{G M_{\text {baryon }}}{r^{2}}
$$

Again, we assume spherical symmetry for the calculation of total mass. The acceleration was determined at the same radii for which the temperature and density values were available. These radii are not the same for all the groups used in our analysis. We used a total of 178 data points for all the groups.

\section{ANALYSIS AND RESULTS}

We now discuss our results on the constancy of the halo surface density and RAR.

\section{A. Determination of scaling relations}

The $\rho_{c}$ and $r_{c}$ values along with the $1 \sigma$ errors for the sample of 17 groups determined by fitting the density profiles to Eq. 8, as prescribed in Section II A are tabulated in Table I. The core density and core radius are plotted on log-log scale in Figure 1. We find (similar to previous works) that the core density is inversely proportional to the core radius. We now determine the scaling relation between $\rho_{c}$ and $r_{c}$ in the same way as in GD20, by fitting for $y=m x+b$, where $y \equiv \ln \left(\rho_{c}\right)$ and $x \equiv \ln \left(r_{c}\right)$.

We estimated the best-fit parameters of $m$ and $b$ by maximizing the logarithm of likelihood function given by:

$$
\begin{aligned}
-2 \ln L & =\sum_{i} \ln 2 \pi \sigma_{i}^{2}+\sum_{i} \frac{\left[y_{i}-\left(m x_{i}+b\right)\right]^{2}}{\sigma_{i}^{2}} \\
\sigma_{i}^{2} & =\sigma_{y_{i}}^{2}+m^{2} \sigma_{x_{i}}^{2}+\sigma_{\text {int }}^{2}
\end{aligned}
$$




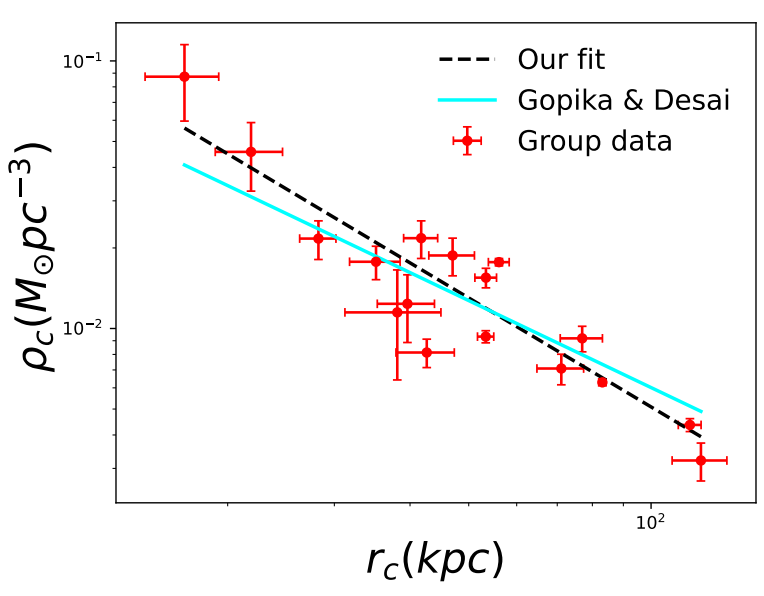

FIG. 1: $\rho_{c}$ versus $r_{c}$ for the Z06 and G07 group sample.

The black dashed line represents the fitted line from this analysis $\left(\rho_{c} \propto r_{c}^{-1.35}\right)$. The cyan line shows the fit obtained for the clusters analyzed in GD20 [1].

An unknown intrinsic scatter $\left(\sigma_{\text {int }}\right)$, considered as a free parameter, is added in quadrature to the errors in $x$ $\left(\sigma_{x}\right)$ and $y\left(\sigma_{y}\right)$, similar to our previous works [1, 36, 53].

The maximization of the likelihood is done using the emcee MCMC sampler [54]. The best-fit value for the scaling relation between $\rho_{c}$ and $r_{c}$, which we obtained is given by:

$$
\begin{array}{r}
\ln \left(\frac{\rho_{c}}{M_{\odot} p c^{-3}}\right)=\left(-1.35_{-0.17}^{+0.16}\right) \ln \left(\frac{r_{c}}{k p c}\right) \\
+\left(0.94_{-0.68}^{+0.66}\right)
\end{array}
$$

with an intrinsic scatter of $\left(27.3_{-8.9}^{+9.2}\right) \%$. The best fit values and their $1 \sigma$ levels for the slope $(m)$, intercept $(b)$ are superposed in Figure 2 along with the data. We find that the intrinsic scatter for this scaling relation is about a factor of 1.5 times larger than what was seen for the Chandra cluster sample analyzed in GD20. Therefore, the relation between $\rho_{c}$ and $r_{c}$ is not as tight as seen for galaxy clusters. We find a deviation from a constant dark matter halo density at about $2.0 \sigma$. We also redid the analysis by omitting the RGH 80 group data (given that it may not be in hydrostatic equilibrium), and obtained a slope of $-1.40_{-0.17}^{+0.18}$ with an intrinsic scatter of $27.3 \%$. Therefore, we find that this group does not make an appreciable difference to the final results.

Finally, the median value of $S_{\text {surf }}$ for our sample is equal to $612 M_{\odot} / p c^{2}$, which is about four times larger than the values for single galaxies [6], but still smaller than what was obtained for galaxy clusters in GD20. Therefore, this shows that the halo surface density cannot be a constant across all scales.

\begin{tabular}{ccc}
\hline \hline Galaxy Group & $\begin{array}{c}\rho_{c} \\
10^{-3} M_{\odot} p c^{-3}\end{array}$ & $\begin{array}{r}r_{c} \\
\mathrm{kpc}\end{array}$ \\
\hline IC 1860 & $9.33 \pm 0.49$ & $53.34 \pm 1.65$ \\
A262 & $15.49 \pm 1.30$ & $53.38 \pm 2.19$ \\
A2717 & $4.36 \pm 0.24$ & $115.92 \pm 5.02$ \\
AWM 4 & $9.19 \pm 1.01$ & $76.96 \pm 6.16$ \\
ESO 3060170 & $17.70 \pm 0.56$ & $56.10 \pm 2.22$ \\
ESO 5520200 & $3.21 \pm 0.52$ & $120.87 \pm 12.54$ \\
MKW 4 & $21.76 \pm 3.49$ & $41.73 \pm 2.70$ \\
MS 0116.3-0115 & $7.09 \pm 0.93$ & $71.09 \pm 6.29$ \\
NGC 533 & $12.37 \pm 3.50$ & $39.61 \pm 4.29$ \\
NGC 1550 & $45.72 \pm 13.10$ & $21.86 \pm 2.78$ \\
NGC 2563 & $11.49 \pm 5.07$ & $38.11 \pm 6.88$ \\
NGC 4325 & $17.76 \pm 2.54$ & $35.15 \pm 3.36$ \\
NGC 5129 & $8.13 \pm 0.99$ & $42.63 \pm 4.71$ \\
RGH 80 & $21.68 \pm 3.57$ & $28.24 \pm 1.94$ \\
RX J1159.8+5531 & $18.75 \pm 3.01$ & $47.03 \pm 4.07$ \\
NGC 5044 & $87.32 \pm 27.73$ & $16.97 \pm 2.36$ \\
A2589 & $6.29 \pm 0.15$ & $83.11 \pm 1.15$ \\
\hline \hline
\end{tabular}

TABLE I: Estimated values for the core density $\left(\rho_{c}\right)$ and the core radius $\left(r_{c}\right)$ for the Z06 and G07 galaxy group sample. The median value of their product, $S_{\text {surf }}$ is equal to $612 M_{\odot} / p c^{2}$.

\section{RADIAL ACCELERATION RELATION IN GROUPS}

We have also used this same dataset to implement a test for the RAR, similar to the analysis in [36] (P21, hereafter). The estimated total and baryonic acceleration for the radii for which the data and errors were available to us was stacked together. A plot showing the relation between the two in the logarithmic space can be found in Figure 3.

To test the RAR, a linear regression was done by maximizing the log-likelihood function (Eq. 11) in the same way as was done in the previous sub-section. Here, $x \equiv \ln \left(a_{\text {tot }}\right)$ and $y \equiv \ln \left(a_{\text {baryon }}\right)$, and their errors are denoted by $\sigma_{x}$ and $\sigma_{y}$, respectively as before. We again incorporate an intrinsic scatter as a free parameter. We obtained $a_{\text {tot }} \propto a_{\text {baryon }}^{0.36 \pm 0.02}$ with an intrinsic scatter of $42 \%$. The best-fit results are tabulated in Table II, and can be compared with the results from other cluster data. The $68 \%, 90 \%$, and $99 \%$ marginalized confidence intervals for $m, b$, and $\sigma_{\text {intrinsic }}$ can be found in Fig. 4 .

The residual scatter in the RAR for this group sample is $\sigma=0.32$ dex. Similar to P21, this was obtained by fitting a Gaussian function to the frequency distribution of the difference between $\log _{10}\left(a_{o b s}\right)$ and $\log _{10}\left(a_{\text {expected }}\right)$. The standard deviation of the fitted Gaussian function gives the residual scatter in dex, which is used to characterize the tightness of RAR relation of this sample. As we can see, the residual scatter obtained (0.32 dex) is 


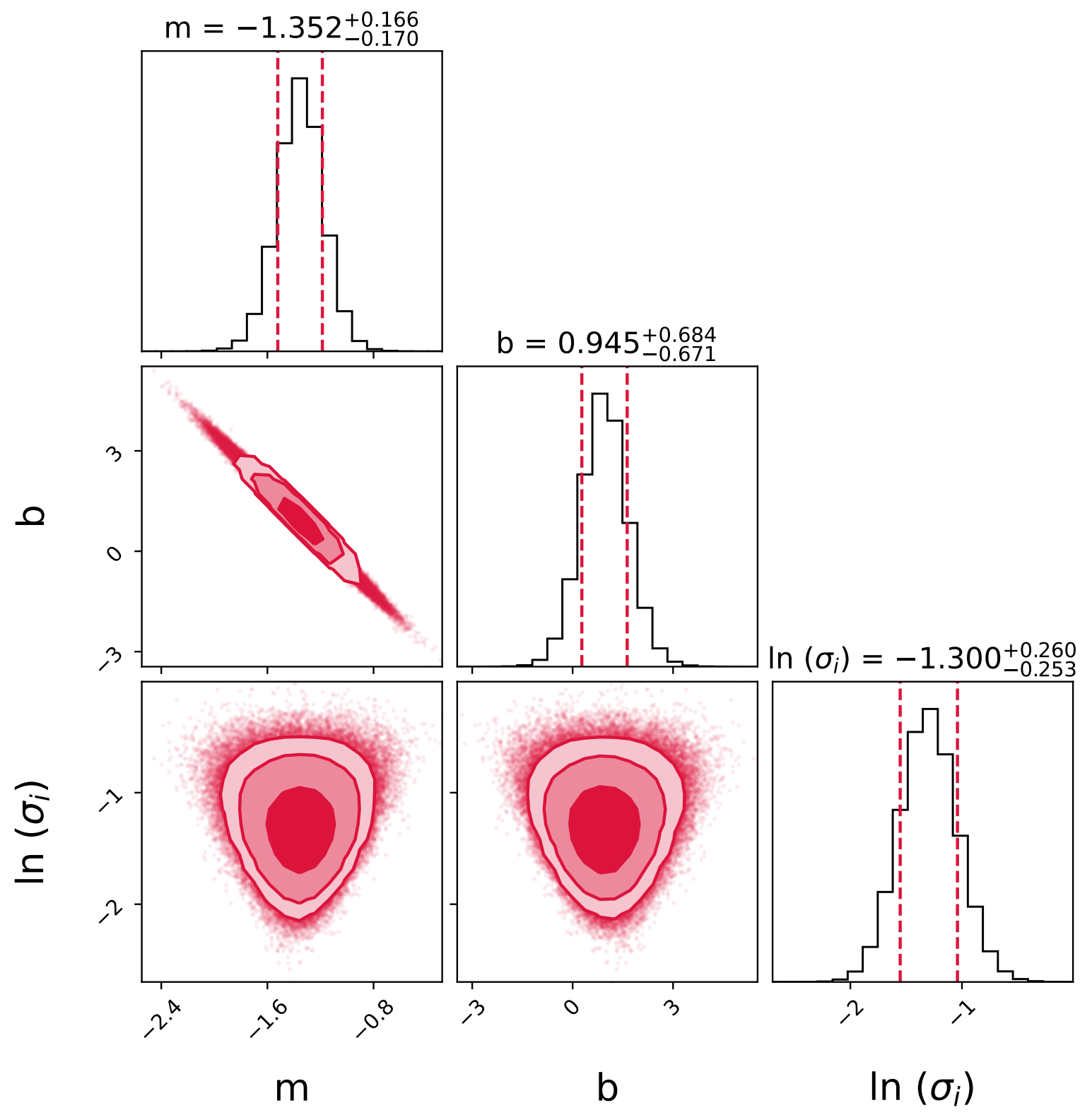

FIG. 2: Plot showing the $68 \%, 90 \%$ and $99 \%$ marginalized credible intervals for $m$ (slope), $b$ (intercept), and $\ln \sigma_{i}$ (intrinsic scatter) for the linear relation between $\rho_{c}$ and $r_{c}$ in $\log -\log$ space.

\begin{tabular}{lccccc}
\hline \hline Slope & Intercept & Intrinsic Scatter & Residual Scatter(dex) & $a_{0}\left(\mathrm{~m} / \mathrm{s}^{2}\right)$ & Data Sample \\
\hline $0.36_{-0.02}^{+0.02}$ & $-13.87_{-0.45}^{+0.45}$ & $0.42 \pm 0.02$ & 0.32 & $(0.88 \pm 0.06) \times 10^{-9}$ & XMM and Chandra (this work) \\
$1.09_{-0.07}^{+0.07}$ & $4.21_{-0.1}^{+0.1}$ & $1.36 \pm 0.02$ & 0.11 & $(1.12 \pm 0.11) \times 10^{-9}$ & XCOP[36] \\
$0.51_{-0.05}^{+0.04}$ & $-10.17_{-0.03}^{+0.03}$ & $0.49 \pm 0.02$ & 0.11 & $(2.02 \pm 0.11) \times 10^{-9}$ & CLASH[26] \\
\hline \hline
\end{tabular}

TABLE II: Summary of results for a linear regression of $\ln \left(a_{t o t}\right)$ versus $\ln \left(a_{\text {baryon }}\right)$ from different group and cluster samples. 


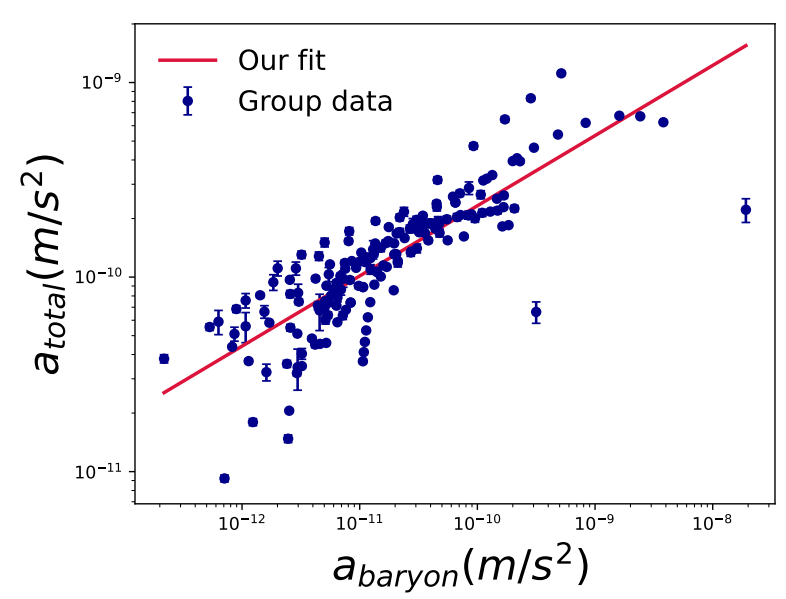

FIG. 3: A test of the RAR using the 17 groups from Z06 and G07 sample. The crimson solid line is the fit from our analysis.

about three times higher than what was obtained with the cluster samples [26, 36, 37] or for galaxies [55]. Therefore, we conclude that our sample of galaxy groups does not show the same tight scatter seen previously for other astrophysical systems.

The acceleration scale $\left(a_{0}\right)$ is evaluated in the same way as in P20 (See also [26]). The slope for the linear regression is fixed to 0.5 in the regression relation, thereby recasting the RAR relation as $a_{d y n}=\sqrt{a_{0} a_{\text {baryon }}}$. The best-fit value with this assumption for $a_{0}$ is $(0.88 \pm 0.06) \times$ $10^{-9} \mathrm{~m} / \mathrm{s}^{2}$, which is slightly lower than what was obtained for CLASH [26] and XCOP [36] data, but still larger than what was seen for the SPARC sample [23].

\section{CORRELATION WITH MASS AND LUMINOSITY}

We now use our estimates for $S_{\text {surf }}$ to test for any putative correlation with mass and luminosity, which have been reported using observational data on galactic scales $[4,18,21]$, and also to test the predictions of some theoretical models $[18,45]$. We carry out a regression analysis in log-log space between each of the observables. A summary of these results along with the intrinsic scatter for each of these relations is summarized in Table III.

To check for a correlation of $S_{\text {surf }}$ with the halo mass, similar to GD20, we use $M_{200}$ as a proxy, where $M_{200}$ is the mass at the over-density $\Delta=200$ with respect to the critical density. $M_{200}$ values were available in G07 and Z06, which were obtained through NFW [56] fits. The dark matter surface density $(S)$ for each of the groups as a function of $M_{200}$ is shown in Figure 5. While testing for a correlation between the two, we incorporated the cluster data from GD20 in order to increase the dynamical range for the mass. A marginal positive correlation $\left(S \propto M_{200}^{0.11 \pm 0.07}\right)$, can be seen between these observ- ables, which is in accord with some of the previous results seen at galactic scales $[18,21]$. However, additional data is necessary to confirm if this correlation is significant. From Figure 5, we also find that the dark matter surface density of groups can be delineated from that of clusters and falls in the left corner of the plot.

Previously, contradictory results have been obtained for the correlation between halo surface density and luminosity (or magnitude). The analysis in $[3,4]$ found no correlation between the surface density and $B$-band absolute magnitude. However, most recently, Zhou et al. (see also [20]) showed that for the SPARC sample, the surface density is correlated with the luminosity measured at $3.6 \mu \mathrm{m}$. Our data for $S$ as a function of $L_{K}$ is shown in Fig. 6. We find that $S \propto L_{k}^{-0.08 \pm 0.19}$. Therefore, although we find that there is no evolution of $S$ with $L_{k}$ within $1 \sigma$, we also cannot rule out any correlation (or anti-correlation) with the luminosity. Additional data for group scale haloes is necessary to make a definitive statement.

Previously, a correlation between $r_{c}$ and the stellar mass was found, using the $B$-band luminosity $\left(L_{B}\right)$ as a proxy [4]. Their analysis showed that $r_{c} \propto L_{B}^{0.446}$. Here, since we are using $L_{K}$ as a proxy for stellar mass, we check for correlation between $r_{c}$ and $L_{K}$. This data is shown in Fig. 7. There is a slight positive correlation between these quantities, $r_{c} \propto L_{K}^{0.71 \pm 0.2}$, in agreement with these results.

We should also caution that the intrinsic scatter for all the aforementioned relations (cf. Table III) is also quite high $>30 \%$. Therefore, for a more definitive test, much more data would be needed to ascertain some of these correlations and check if the scatter becomes tighter with more statistics.

\section{CORRELATION OF DARK COLUMN DENSITY WITH MASS}

We now calculate a variant of the dark matter surface density called dark matter "column density", for a dark matter density profile $\left(\rho_{D M}\right)$ defined at radius $(R)[15$, $57]$

$$
S(R)=\frac{2}{R^{2}} \int_{0}^{R} r^{\prime} d r^{\prime} \int_{-\infty}^{+\infty} d z \rho_{D M}\left(\sqrt{r^{\prime 2}+z^{2}}\right)
$$

This measure of surface density is more robust than $S_{\text {surf }}$, as it has been shown to be invariant with respect to the underlying dark matter density profile used $[15,18]$. Therefore, this column density has also been used as an alternative probe of average dark matter surface density in literature. The column densities for NFW $\left(S_{N F W}\right)$, pseudo-isothermal $\left(S_{I S O}\right)$ and Burkert profiles $\left(S_{B U R}\right)$ are linked with each other according to : $S_{N F W}\left(r_{s}\right) \approx$ $0.91 S_{I S O}\left(6 r_{c}\right)$ and $S_{N F W}\left(r_{s}\right) \approx 0.98 S_{B U R}\left(1.66 r_{0}\right)$ [15]. The analytical expressions for $S_{N F W}$ and $S_{I S O}$ have been provided in $[15,18]$. 


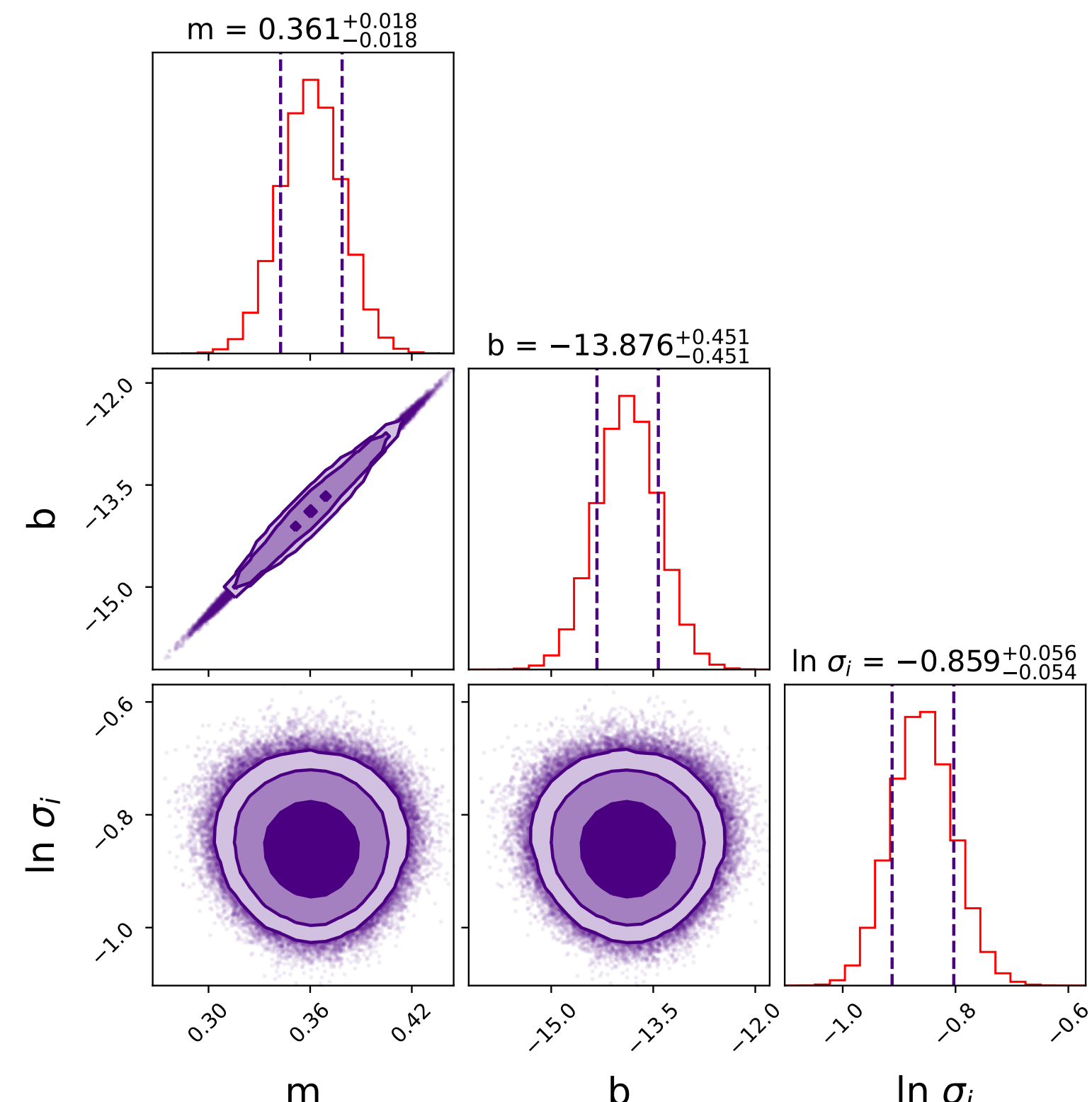

FIG. 4: Plot showing the $68 \%, 90 \%$ and $99 \%$ marginalized credible intervals for $m$ (slope), $b$ (intercept), and $\ln \sigma_{i}$ (intrinsic scatter) for the linear relation between $a_{t o t}$ and $a_{b a r y o n}$ in $\log -\log$ space.

\begin{tabular}{lccc}
\hline \hline Slope & Intercept & Intrinsic Scatter(\%) & Scaling Relation \\
\hline $0.11_{-0.07}^{+0.07}$ & $3.0_{-2.42}^{+2.43}$ & $35.5_{-5.9}^{+5.5}$ & $S_{\text {surf }}-M_{200}$ \\
$-0.08_{-0.19}^{+0.19}$ & $8.61_{-5.30}^{+5.14}$ & $32.5_{-9.0}^{+8.6}$ & $S_{\text {surf }}-L_{k}$ \\
$0.71_{-0.19}^{+0.20}$ & $-15.39_{-5.4}^{+5.2}$ & $39.1_{-6.4}^{+7.5}$ & $r_{c}-L_{k}$ \\
$0.13_{-0.09}^{+0.09}$ & $1.39_{-3.14}^{+2.92}$ & $24.8_{-7.7}^{+8.1}$ & $S_{\text {Bur }}-M_{200}$ \\
\hline \hline
\end{tabular}

TABLE III: Summary of the study of possibly correlation of the halo surface density $(S)$ and core radius $\left(r_{c}\right)$ with mass and luminosity. In each of these cases we fit the observables using linear regression in log-log space 


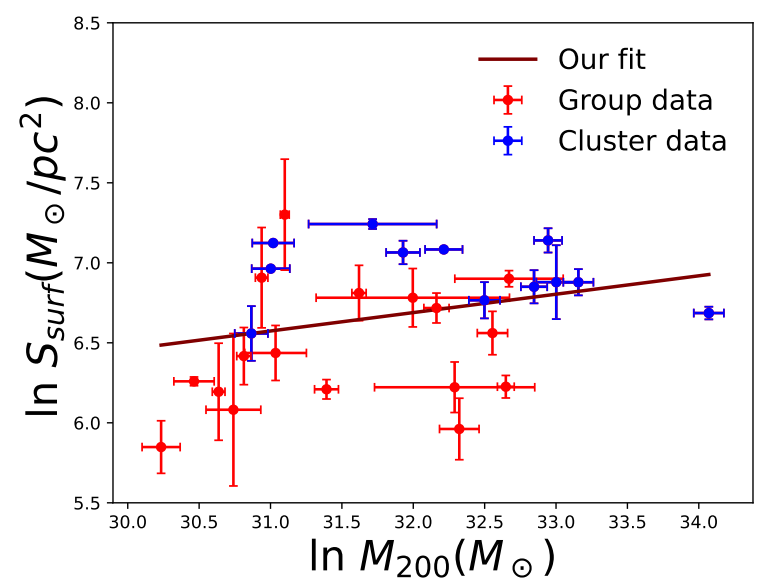

FIG. 5: Correlation of dark matter halo surface density with mass using data for galaxy groups analyzed in this work and galaxy clusters from GD20. The black solid line shows the fit from our analysis. The best-fit values can be found in Table III.

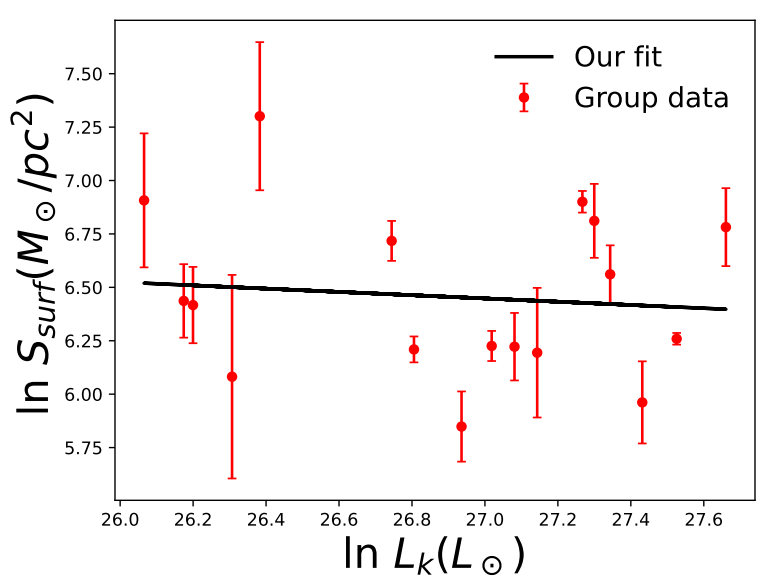

FIG. 6: Correlation of halo surface density with $K$-band luminosity. The best-fit values can be found in

Table III.

The values of $S_{N F W}$ for all the galaxy groups analyzed in this work have been tabulated in [15], who find that $S_{N F W} \propto M_{\text {halo }}^{0.2}$. Here, we calculate $S_{B U R}$ using the $\rho_{c}$ and $r_{c}$ data obtained for each group from Table I. $S_{B U R}$ was calculated by plugging in the Burkert density profile in Eq. 13, and numerically evaluating the integrals. The values of $S_{B U R}$ for all the groups of our sample are plotted as a function of $M_{200}$ in Fig. 8. $S_{B U R}$ is of the same order of magnitude as $S_{\text {surf }}$, with their median ratio equal to 0.71 . We carry out a regression analysis in the same way as in Sect. V. Our best-fit values are tabulated in Table III. Similar to $S_{\text {surf }}$, we find a mild correlation with halo mass $\left(S_{\text {burk }} \propto M_{200}^{0.13 \pm 0.09}\right)$. However, the intrinsic scatter is about $25 \%$, which although is slightly smaller than that obtained for the halo surface

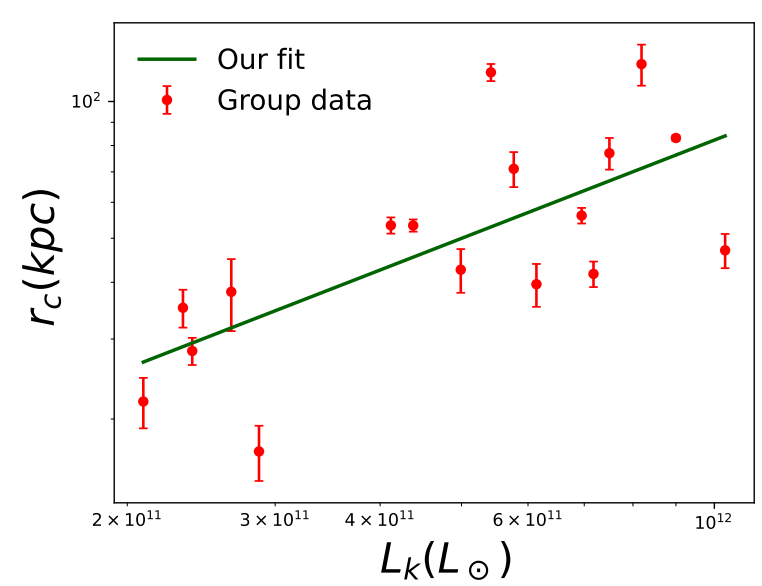

FIG. 7: Correlation of $K$-band luminosity with the halo core radius. The best-fit values can be found in

Table III.

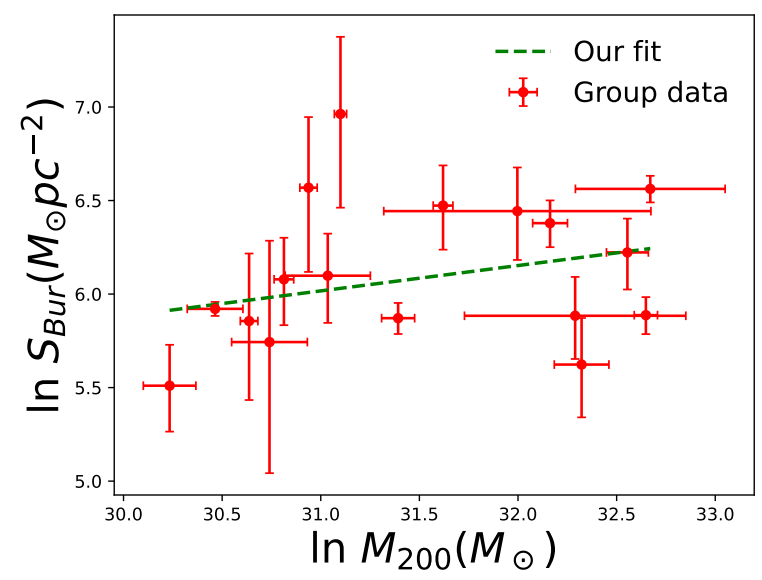

FIG. 8: Correlation of the dark matter column density calculated for the Burkert profile (cf. Eq. 13) with halo mass. The best-fit values can be found in Table III.

density is still quite large.

\section{CONCLUSIONS}

In this work, we implemented a test for the constancy of the dark matter halo surface density as well as column density and RAR for a sample of 17 galaxy groups observed using Chandra and XMM-Newton.

Several studies done previously with galaxies unveiled a very intriguing result that the dark matter halo surface density, given by the product of $\rho_{c}$ and $r_{c}$, obtained from fitting a cored profile, is a constant irrespective of the mass, spectral type, and blue magnitude [3, 4]. However, this result assumes that dark matter density profiles can be well-fitted by cored profiles, and consequently has been disputed by several other works, which find a corre- 
lation with halo mass. The observed halo surface density has proved to be an invaluable probe of alternatives to the standard $\Lambda$ CDM model $[8,22]$.

Very few studies of this ansatz were previously done with galaxy clusters, apart from a detailed analysis of a ROSAT cluster sample [8], which showed that galaxy clusters do not have a constant surface density. Recently, GD20 carried out a systematic test using a sample of 12 Chandra clusters, and found that $\rho_{c} \propto r_{c}^{-1.08_{-0.06}^{+0.05}}$, indicating a deviation from a constant surface density by about $1.4 \sigma$. They also showed that the halo surface density for the cluster sample is about ten times larger than that for galaxies.

Here, we have extended the same analysis to lower mass systems, using a set of 17 relaxed galaxy groups from XMM-Newton and Chandra X-ray observations. We find that the dark matter core density $\left(\rho_{c}\right)$ and core radius $\left(r_{c}\right)$ obey the scaling relation, $\rho_{c} \propto r_{c}^{-1.35 \pm 0.17}$. However, the intrinsic scatter of $27 \%$ which we obtain is about 1.5 times larger than that seen for clusters, indicating that the relation is not as tight as seen for galaxy clusters. Furthermore, the halo surface density is discrepant with respect to a constant value by about $2.0 \sigma$. The dark matter surface density which we obtained for the groups falls just below the clusters and is about four times larger than that obtained for galaxies [6].

We have also tested for a correlation of both the surface density and column density with mass and obtained a marginal positive correlation (albeit with a large scatter), which is in agreement with some previous estimates [18]. We cannot confirm or rule out a correlation of the surface density with $K$-band luminosity. We also find that the core radius is correlated with $K$-band luminosity, in agreement with the results in [4].

As a follow-up to another recent work of ours which tested the RAR for three different cluster samples [36, 37], we also implemented a test of RAR using the same group sample to see how well RAR holds up at group scales. Our results are summarized in Table II. We find that the residual scatter in the RAR relation for groups is about 0.3 dex, and subsequently about three times larger than that seen for galaxies or clusters. Therefore, galaxy groups do not obey the RAR. The acceleration scale which we obtain assuming a slope of 0.5 , is between the values seen for clusters and single galaxies.

Additional tests for the halo surface density and RAR with additional group catalogs (for eg [41]) and the upcoming e-ROSITA sample, followed by comparison with predictions from $\Lambda \mathrm{CDM}$ and alternatives will be discussed in future works.

Nevertheless, the result in this work in conjunction with G20, prove that the dark matter halo surface density cannot be a universal constant at all scales or for all dark matter dominated systems.

\section{Acknowledgements}

We are grateful to Fabio Gastaldello for useful correspondence and for providing us the data in G07. We would also like to thank Man-Ho Chan, Antonio Del Popolo, Yong Tian, and S. Pradyumna for helpful discussions on this topic. Finally, we thank the anonymous referee for useful comments and feedback on our manuscript. 
[1] K. Gopika and S. Desai, Physics of the Dark Universe 30, 100707 (2020), 2006.12320.

[2] A. Vikhlinin, A. Kravtsov, W. Forman, C. Jones, M. Markevitch, S. S. Murray, and L. Van Speybroeck, Astrophys. J. 640, 691 (2006), astro-ph/0507092.

[3] F. Donato, G. Gentile, P. Salucci, C. Frigerio Martins, M. I. Wilkinson, G. Gilmore, E. K. Grebel, A. Koch, and R. Wyse, Mon. Not. R. Astron. Soc. 397, 1169 (2009), 0904.4054 .

[4] J. Kormendy and K. C. Freeman, Astrophys. J. 817, 84 (2016), 1411.2170.

[5] A. Burkert, Astrophys. J. 808, 158 (2015), 1501.06604.

[6] P. Salucci, Astronomy and Astrophysics Review 27, 2 (2019), 1811.08843.

[7] A. Burkert, Astrophys. J. Lett. 447, L25 (1995), astroph/9504041.

[8] M. H. Chan, Mon. Not. R. Astron. Soc. 442, L14 (2014), 1403.4352 .

[9] J. D. Simon, A. D. Bolatto, A. Leroy, L. Blitz, and E. L. Gates, Astrophys. J. 621, 757 (2005), astro-ph/0412035.

[10] L. E. Strigari, C. S. Frenk, and S. D. M. White, Mon. Not. R. Astron. Soc. 408, 2364 (2010), 1003.4268.

[11] A. Genina, A. Benítez-Llambay, C. S. Frenk, S. Cole, A. Fattahi, J. F. Navarro, K. A. Oman, T. Sawala, and T. Theuns, Mon. Not. R. Astron. Soc. 474, 1398 (2018), 1707.06303 .

[12] M. A. Breddels and A. Helmi, Astron. \& Astrophys. 558, A35 (2013), 1304.2976.

[13] A. B. Newman, T. Treu, R. S. Ellis, D. J. Sand, C. Nipoti, J. Richard, and E. Jullo, Astrophys. J. 765, 24 (2013), 1209.1391.

[14] A. B. Newman, T. Treu, R. S. Ellis, and D. J. Sand, Astrophys. J. 765, 25 (2013), 1209.1392.

[15] A. Boyarsky, O. Ruchayskiy, D. Iakubovskyi, A. V. Maccio', and D. Malyshev, arXiv e-prints arXiv:0911.1774 (2009), 0911.1774.

[16] N. R. Napolitano, A. J. Romanowsky, and C. Tortora, Mon. Not. R. Astron. Soc. 405, 2351 (2010), 1003.1716.

[17] V. F. Cardone and C. Tortora, Mon. Not. R. Astron. Soc. 409, 1570 (2010), 1007.3673.

[18] A. Del Popolo, V. F. Cardone, and G. Belvedere, Mon. Not. R. Astron. Soc. 429, 1080 (2013), 1212.6797.

[19] V. F. Cardone and A. Del Popolo, Mon. Not. R. Astron. Soc. 427, 3176 (2012), 1209.1524.

[20] A. Saburova and A. Del Popolo, Mon. Not. R. Astron. Soc. 445, 3512 (2014), 1410.3052.

[21] Y. Zhou, A. Del Popolo, and Z. Chang, Physics of the Dark Universe 28, 100468 (2020).

[22] A. Burkert, Astrophys. J. 904, 161 (2020), 2006.11111.

[23] S. S. McGaugh, F. Lelli, and J. M. Schombert, Phys. Rev. Lett. 117, 201101 (2016), 1609.05917.

[24] F. Lelli, S. S. McGaugh, J. M. Schombert, H. Desmond, and H. Katz, Mon. Not. R. Astron. Soc. 484, 3267 (2019), 1901.05966.

[25] K.-H. Chae, M. Bernardi, H. Domínguez Sánchez, and R. K. Sheth, Astrophys. J. Lett. 903, L31 (2020), 2010.10779 .

[26] Y. Tian, K. Umetsu, C.-M. Ko, M. Donahue, and I. N. Chiu, Astrophys. J. 896, 70 (2020), 2001.08340.

[27] M. H. Chan and A. Del Popolo, Mon. Not. R. Astron. Soc. p. 218 (2020), 2001.06141.
[28] D. C. Rodrigues, V. Marra, A. Del Popolo, and Z. Davari, Nature Astronomy 2, 927 (2018), 1811.05882.

[29] D. C. Rodrigues, V. Marra, A. Del Popolo, and Z. Davari, Nature Astronomy 4, 134 (2020), 2002.01970.

[30] C. Stone and S. Courteau, Astrophys. J. 882, 6 (2019), 1908.06105

[31] Z. Chang and Y. Zhou, Mon. Not. R. Astron. Soc. 486, 1658 (2019), 1812.05002.

[32] B. Famaey and S. S. McGaugh, Living Reviews in Relativity 15, 10 (2012), 1112.3960.

[33] A. Paranjape and R. K. Sheth, Mon. Not. R. Astron. Soc. (2021), 2102.13116.

[34] L. S. The and S. D. M. White, Astron. J. 95, 1642 (1988).

[35] S. Seeram and S. Desai, Journal of Astrophysics and Astronomy 42, 3 (2021).

[36] S. Pradyumna, S. Gupta, S. Seeram, and S. Desai, Physics of the Dark Universe 31, 100765 (2021), 2011.06421.

[37] S. Pradyumna and S. Desai, Physics of the Dark Universe 33, 100854 (2021), 2107.05845.

[38] M. Sun, New Journal of Physics 14, 045004 (2012), 1203.4228 .

[39] V. R. Eke, C. M. Baugh, S. Cole, C. S. Frenk, P. Norberg, J. A. Peacock, I. K. Baldry, J. Bland-Hawthorn, T. Bridges, R. Cannon, et al., Mon. Not. R. Astron. Soc. 348, 866 (2004), astro-ph/0402567.

[40] A. Finoguenov, T. J. Ponman, J. P. F. Osmond, and M. Zimer, Mon. Not. R. Astron. Soc. 374, 737 (2007), astro-ph/0611846.

[41] M. Sun, G. M. Voit, M. Donahue, C. Jones, W. Forman, and A. Vikhlinin, Astrophys. J. 693, 1142 (2009), 0805.2320.

[42] H. J. Eckmiller, D. S. Hudson, and T. H. Reiprich, Astron. \& Astrophys. 535, A105 (2011), 1109.6498.

[43] A. Morandi, M. Sun, J. Mulchaey, D. Nagai, and M. Bonamente, Mon. Not. R. Astron. Soc. 469, 2423 (2017).

[44] G. W. Angus, B. Famaey, and D. A. Buote, Mon. Not. R. Astron. Soc. 387, 1470 (2008), 0709.0108.

[45] H. W. Lin and A. Loeb, JCAP 2016, 009 (2016), 1506.05471.

[46] F. Gastaldello, D. A. Buote, P. J. Humphrey, L. Zappacosta, J. S. Bullock, F. Brighenti, and W. G. Mathews, Astrophys. J. 669, 158 (2007), astro-ph/0610134.

[47] L. Zappacosta, D. A. Buote, F. Gastaldello, P. J. Humphrey, J. Bullock, F. Brighenti, and W. Mathews, Astrophys. J. 650, 777 (2006), astro-ph/0602613.

[48] A. Mahdavi, A. Finoguenov, H. Böhringer, M. J. Geller, and J. P. Henry, Astrophys. J. 622, 187 (2005), astro$\mathrm{ph} / 0502362$.

[49] V. Biffi, S. Borgani, G. Murante, E. Rasia, S. Planelles, G. L. Granato, C. Ragone-Figueroa, A. M. Beck, M. Gaspari, and K. Dolag, Astrophys. J. 827, 112 (2016), 1606.02293.

[50] S. W. Allen, A. E. Evrard, and A. B. Mantz, Ann. Rev. Astron. Astrophys. 49, 409 (2011), 1103.4829.

[51] P. Kroupa, Mon. Not. R. Astron. Soc. 322, 231 (2001), astro-ph/0009005.

[52] P. J. Humphrey, D. A. Buote, F. Gastaldello, L. Zappacosta, J. S. Bullock, F. Brighenti, and W. G. Mathews, Astrophys. J. 646, 899 (2006), astro-ph/0601301. 
[53] K. Bora and S. Desai, JCAP 2021, 012 (2021), 2008.10541.

[54] D. Foreman-Mackey, D. W. Hogg, D. Lang, and J. Goodman, PASP 125, 306 (2013), 1202.3665.

[55] P. Li, F. Lelli, S. McGaugh, and J. Schombert, Astron. \& Astrophys. 615, A3 (2018), 1803.00022.
[56] J. F. Navarro, C. S. Frenk, and S. D. M. White, Astrophys. J. 490, 493 (1997), astro-ph/9611107.

[57] A. Boyarsky, A. Neronov, O. Ruchayskiy, and I. Tkachev, Phys. Rev. Lett. 104, 191301 (2010), 0911.3396. 